\title{
Epigenetics and transgenerational inheritance
}

\author{
Emilie Brasset ${ }^{1 *}$ and Séverine Chambeyron ${ }^{2 *}$
}

\section{Abstract \\ A report on the 'Non-coding RNA, epigenetics and transgenerational inheritance' meeting, Churchill College, Cambridge, UK, 11-12 April 2013.}

The discovery of RNA interference in 1998 by Andrew Fire and Craig Mello revealed the complexity of gene regulation. The role of non-coding RNAs in post-transcriptional gene silencing is well documented from plants to vertebrates; conversely, their involvement in transcriptional gene silencing by altering chromatin structure is well described in plants and Schizosaccharomyces pombe but has just started to be explored in metazoans. The goal of this meeting was to bring together scientists working on the biogenesis of small non-coding RNAs and on their function as carriers of epigenetic information in fungi, plants and animals. Here, we highlight the four major themes discussed at the meeting: the wide variety of biological functions undertaken by small non-coding RNAs; the role of Piwi-interacting RNAs (piRNAs) in RNA biogenesis and transposable element silencing; the roles of small non-coding RNAs in intercellular and systemic signaling; and transgenerational epigenetic inheritance.

\section{Piwi-interacting RNA biogenesis and transposable element silencing}

It is well established that piRNAs bind Piwi proteins, which are members of the Argonaute family, and are employed in the silencing of transposable elements (TEs). However, the mode of action and the function of the piRNA pathway proteins remains elusive, possibly because of their specific expression in the gonads, which makes piRNAs more difficult to study.

\footnotetext{
*Correspondence: emilie.brasset@udamail.fr; severine.chambeyron@igh.cnrs.fr 'GReD - Inserm, U1103, CNRS UMR6293, Clermont Université, Université d'Auvergne, Faculté de Médecine, 28 place Henri Dunant, 63001 Clermont-Ferrand Cedex, France

Institut de Génétique Humaine, Centre National de la Recherche Scientifique, 34396 Montpellier Cedex 5, France
}

piRNAs are produced by two mechanisms: the primary processing pathway and secondary biogenesis, which is also called the ping-pong amplification loop. Primary piRNAs are likely processed from single-stranded precursor transcripts that are encoded by heterochromatic loci called piRNA clusters. Secondary piRNAs result from the processing of transposon mRNAs and piRNA clusters by an efficient feed-forward amplification loop. Mutation of piRNA pathway components in several organisms (Bombyx, Drosophila, mice and zebrafish) has provided a better understanding of their functions in the piRNA biogenesis pathways.

Ramesh Pillai (European Molecular Biology Laboratory, Grenoble, France) showed in both Bombyx mori and Drosophila melanogaster that the DEAD box domain of an RNA helicase, Vasa, is required for disassembling the ribonucleoprotein complex during secondary biogenesis of piRNAs. He also reported that a mouse Tudordomain-containing protein, TDRD12, is needed for secondary biogenesis. Emile Brasset (Auvergne University, Clermont-Ferrand, France) reported differences in the spatio-temporal requirement of piRNA pathway components for TE silencing during Drosophila oogenesis using a sensor transgene based on the Idefix transposon. Moreover, in the dividing cysts of the germarium, a component of insect ovaries, she described a time window that she called the 'piwiless pocket', when low piwi expression correlates with loss of repression of the Idefix sensor.

To go further in understanding the piRNA pathway in zebrafish, René Ketting (Hubrecht Institute, the Netherlands) studied Tdrd6, a newly identified component of the piRNA pathway. This protein seems to be required for the correct localization of the Argonaute-family protein Ziwi to the nuage, a perinuclear cytoplasmic structure, in embryos, but not for piRNA biogenesis, as only a minor piRNA loss was observed in mutants. Tdrd6 was also reported to be expressed in the ovarian ooplasm's Balbiani body, a transient cytoplasmic structure composed of various organelles. He proposed that Tdrd6 might be involved in maternal transcript inheritance.

During spermatogenesis in mice, primordial germ cells undergo massive epigenetic reprogramming as they colonize the fetal gonads, notably in the form of a genome-wide loss of DNA methylation, which correlates 
with TE expression. Fetal male germ cells specifically express the Argonaute-family proteins Mili and Miwi2, which are responsible for the production of piRNAs through the degradation of transposon mRNAs. Donal O'Carroll (EMBL, Monterotondo, Italy) described a conditional mili mutant in which slicer activity, which is required for the secondary piRNA processing mechanism, was substantially reduced in the pre-leptotene to zygotene stages. He demonstrated that Mili slicer activity is not required for the repression of the $\mathrm{L} 1$ retrotransposon during this time window. Transposon silencing is maintained by deposition of the H3K9me2 histone mark, which is lost at the zygotene transition. He showed that L1 silencing requires the orchestration of three distinct mechanisms during spermatogenesis: DNA methylation, histone methylation and post-transcriptional silencing by piRNAs.

\section{Small non-coding RNAs: a wide repertoire of biological function}

In $S$. pombe, non-coding RNAs are involved in the formation of constitutive heterochromatin at pericentromeric regions by orchestrating the deposition of heterochromatin marks. However, to maintain genome homeostasis heterochromatin and euchromatin must be partitioned; centromeric heterochromatin is demarcated from euchromatin by transcriptionally active tRNA genes. Marc Bühler (Friedrich Miescher Institute, Basel, Switzerland) described a new class of small non-coding RNAs that are produced at the edge of heterochromatin. He suggested that their role was to counteract the spreading of heterochromatic regions that do not have tRNA sequences. These new classes of 22-nucleotidelong RNAs are not loaded onto Argonaute proteins, and they accumulate in dicer 1 mutants.

Eric Miska (Gurdon Institute, University of Cambridge, UK) and Scott Kennedy (Laboratory of Genetics, Madison, USA) reported the role of piRNAs in the regulation of germinal transcripts in Caenorhabditis elegans. Using a transcriptomic approach Miska's team showed that the transgenerational sterility observed when the piRNA pathway is abolished in mutants in the Argonaute-family gene prg-1 is not due to loss of genome integrity but rather to accumulation of toxic transcripts derived from short repeats. The presence of these transcripts in the progeny of $p r g-1$ mutant worms caused epigenetic stress leading to sterility. Surprisingly, this phenotype could be rescued by treatment with insulin, although the underlying mechanism is not known.

Kennedy and colleagues observed that, in the absence of the nuclear RNAi machinery, nematodes become sterile due to defects in gamete formation and function that become progressively worse over generations. Using a genetic screen, they identified the Argonaute protein
HRDE-1 as an actor of multigenerational RNAi inheritance that is essential to maintain the germ-cell lineage. In their model, the deficiency in the RNAi machinery could be correlated with a slow decrease of the H3K9me3 repressive chromatin mark during several generations. Consequently, potentially toxic regions or genes may be transcribed over time, thus destabilizing germline stem cells.

\section{Small non-coding RNAs in intercellular and systemic signaling}

Studies in plants, fission yeast and worms suggest that RNA silencing requires mobile RNA regulators that move from cell to cell, and long distance silencing has been shown in plants by grafting plants of one genotype onto another. In Arabidopsis thaliana, long distance cell to cell mobility has been reported for various types of small non-coding RNAs, notably the 21- and 24-nucleotide RNA species implicated in post-transcriptional regulation and transcriptional silencing, respectively. Using a potato grafting model, David Baulcombe (University of Cambridge, UK) demonstrated that production of 24-nucleotide small RNAs in the shoot can cause transmissible transcriptional gene silencing of a target expressed in the tuber. This silencing was associated with promoter methylation in the grafted potatoes, indicating that the epigenome of a plant can be altered to create epigenetically modified plants. Grafting might be a new tool for transferring an epigenetic modifier (specifically, a small RNA) and thus to establish transcriptional silencing by DNA methylation.

The suggestion of a role for mobile small non-coding RNAs in gene silencing is also strengthened by work in nematodes. Miska and colleagues have addressed the fundamental question of the possible functions for RNA interference in the life cycle of nematodes in the wild. Several clades of parasitic nematodes, including Brugia malayi, live in symbiosis with intracellular bacteria such as Wolbachia, whereas others, for instance Globodera pallida or Trichinella spiralis, are parasites of plants and animals. By analyzing the small RNA populations of parasitic nematodes, they found that small RNA pathways are conserved between $C$. elegans and parasitic nematodes. Furthermore, this analysis revealed the presence of host-parasite communication mediated by small RNAs, suggesting that RNA interference may be important for adapting to the environment. The best example is the intramuscular form of the vertebrate parasite $T$. spiralis, which selectively secretes a miRNA that is homologous to a mammalian miRNA, which then represses muscle differentiation. Given the profound effect of T. spiralis on muscle cell biology, they suggested that parasite miRNAs can influence the expression of host genes. 


\section{Transgenerational epigenetic inheritance}

Since the first evidence of transgenerational epigenetic inheritance in plants, the idea has been put forward that epigenetic marks, such as DNA methylation, histone modifications and small RNAs, could contribute to transgenerational inheritance of acquired traits in metazoans as well. Several presentations reported advances in our understanding of transgenerational epigenetic inheritance. Stephane Ronsseray (UMR7622, CNRS-Paris, France) described the first case of paramutation in insects. Paramutation is the phenomenon in which one allele at a locus induces a heritable epigenetic change of a homologous allele. He has studied a Drosophila homologydependent silencing mechanism used by the P element transposon to establish its repression, and has found that a stable paramutation (over 70 generations) can be induced simply by maternal inheritance of a cytoplasm that carries piRNAs homologous to the paramutated locus. In order to propagate the phenomenon, the paramutated locus itself becomes a strong producer of piRNAs and is fully paramutagenic. Importantly, paramutation in Drosophila can be induced exclusively by cytoplasmic inheritance and does not require pairing between the paramutagenic and paramutated loci. Séverine Chambeyron (Institute of Human Genetics, Montpellier, IGH, France) showed that the ability to repress the I element retrotransposon in the Drosophila female germline behaves like a chromatin-independent acquired epigenetic trait that is exclusively transmitted over generations by the piRNAs that are maternally deposited in the Drosophila embryo.

In mammals, environmental changes, stress and diet may cause a wide range of metabolic and cellular disorders that can be transmitted through several generations. The challenge is to demonstrate that the transmitted traits are indeed epigenetic traits and not a consequence of heritable genetic changes. Erica Watson (University of Cambridge, UK) developed an approach based on the mutation of a gene essential for folate metabolism (Mtrr) to analyze the transgenerational effects of abnormal folate metabolism on mammalian development. Mtrr deficiency in the maternal grandparents is sufficient to cause abnormal mouse development (intrauterine growth restriction, developmental delay and congenital malformations) in the wild-type grandprogeny. Embryo transfer experiments performed to eliminate environmental maternal effects indicate epigenetic inheritance as a mechanism, the results of which persist for at least four generations.
Isabelle Mansuy (University of Zurich, Switzerland) developed a mouse model to test whether early postnatal stress contributes to severe behavioral and metabolic impairment in the progeny. By examining the profile of small RNAs in male germ cells of F1 animals she identified an alteration of the expression of several classes of small RNAs. Injection of small RNAs from stressed males into fertilized mouse oocytes reproduced the same behavioral and metabolic symptoms, strongly suggesting that small RNAs could be the molecules responsible for epigenetic inheritance in this system.

Finally, an intergenerational epigenetic inheritance study using a mouse model of prenatal undernutrition or protein-restriction was presented by Anne FergusonSmith (University of Cambridge, UK). In FergusonSmith's model, the F1 and F2 progeny of undernourished animals (F0) showed low birth weight, defects in glucose tolerance, and an impaired glucose-stimulated insulin response. F0 animals were subjected to nutritional restriction during the third week of pregnancy, a period that coincides with the time of re-acquisition of genomewide methylation, including at imprinting control regions in the male primordial germ cells. Changes in DNA methylation of imprinted genes in the F1 and F2 generations were not identified, suggesting that prenatal undernutrition does not perturb the functional monoallelicism of imprinted genes and their mechanisms of epigenetic regulation.

\section{Conclusions}

In recent years, genetic approaches performed in a number of model organisms have been critically important for developing models of the molecular bases for epigenetic regulation of gene expression. These findings highlight the importance of non-coding RNAs and their influence on DNA. The next steps will be to fully characterize the mechanistic details of these processes, and the extent to which they are able to be transmitted over generations.

Competing interests

The authors declare that they have no competing interests.

Acknowledgements

This work was supported by the CNRS, INSERM and Auvergne University.

Published: 24 May 2013

doi:10.1186/gb-2013-14-5-306

Cite this article as: Brasset E, Chambeyron S: Epigenetics and

transgenerational inheritance. Genome Biology 2013, 14:306. 